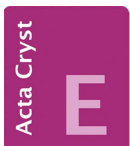

CRYSTALLOGRAPHIC COMMUNICATIONS

ISSN 2056-9890

Received 5 August 2019

Accepted 13 August 2019

Edited by J. Ellena, Universidade de Sâo Paulo, Brazil

Keywords: crystal structure; naphthoquinone; lapachol acetate; synthesis; Hirshfeld analysis.

CCDC reference: 1947085

Supporting information: this article has supporting information at journals.iucr.org/e

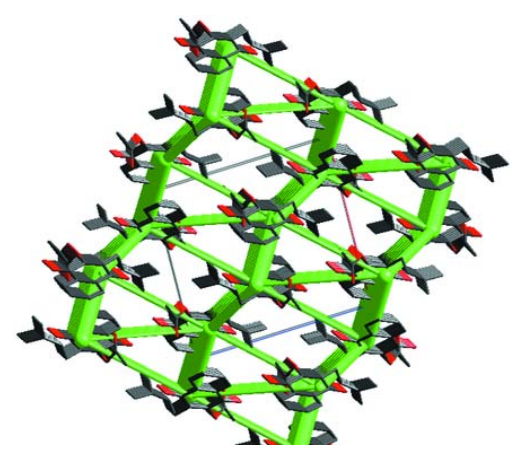

OPEN $\odot$ ACCESS

\section{Crystal structure and Hirshfeld surface analysis of lapachol acetate 80 years after its first synthesis}

\author{
Miguel A. Martínez-Cabrera, ${ }^{a}$ Mario A. Macías, ${ }^{b}$ Francisco Ferreira, ${ }^{c}$ Enrique \\ Pandolfi, ${ }^{d}$ Javier Barúa ${ }^{\mathrm{e}}$ and Leopoldo Suescun ${ }^{\mathrm{f}}$ *
}

\begin{abstract}
a Universidad Nacional de Asunción, Facultad de Ciencias Exactas y Naturales, Departamento de Biología, Área Química Orgánica de los Productos Naturales-LAREV, San Lorenzo Campus-UNA, Paraguay, 'bepartment of Chemistry, Universidad de los Andes, Cra 1 N 18A-12, 111711, Bogotá, Colombia, ' ${ }^{\circ}$ nniversidad Nacional de Asunción, Facultad de Ciencias Exactas y Naturales, Laboratorio de Análisis Instrumental, Departamento de Química, San Lorenzo CampusUNA, Paraguay, 'Laboratorio de Síntesis Orgánica, DQO, Facultad de Química, Universidad de la República, Montevideo 11800, Uruguay, 'e Universidad Nacional de Asunción, Facultad de Ciencias Químicas, San Lorenzo Campus-UNA, Paraguay, and 'Cryssmat-Lab/DETEMA, Facultad de Química, Universidad de la República, Av. Gral. Flores 2124, Montevideo 11800, Uruguay. *Correspondence e-mail: leopoldo@fq.edu.uy
\end{abstract}

Lapachol acetate [systematic name: 3-(3-methylbut-2-enyl)-1,4-dioxonaphthalen-2-yl acetate], $\mathrm{C}_{17} \mathrm{H}_{16} \mathrm{O}_{4}$, was prepared using a modified high-yield procedure and its crystal structure is reported for the first time 80 years after its first synthesis. The full spectroscopic characterization of the molecule is reported. The molecular conformation shows little difference with other lapachol derivatives and lapachol itself. The packing is directed by intermolecular $\pi-\pi$ and $\mathrm{C}-\mathrm{H} \cdots \mathrm{O}$ interactions, as described by Hirshfeld surface analysis. The former interactions make the largest contributions to the total packing energy in a ratio of 2:1 with respect to the latter.

\section{Chemical context}

Naphtoquinones are natural products characterized by a naphthalene ring system exhibiting a para-quinone motif in positions 1,4. They are natural pigments and normally substituted by hydroxyl or methyl groups or present as glycosides (Bruneton, 2001). Among the natural products, they possess remarkable biological activity such as antibacterial, antifungal, antiparasitic, antiviral and anticancer (Babula et al., 2007; da Silva \& Ferreira, 2016; Miranda et al., 2019; Araújo et al., 2019; Barbosa Coitinho et al., 2019; Strauch et al., 2019). Lapachol (2-hydroxy-3-(3-methyl-but-2-enyl)$[1,4]$ naphthoquinone), isolated from Handroanthus Heptaphyllus (Vell.) Mattos, a native species from Paraguay, was studied as a hemisynthetic precursor of lapachol acetate \{[3-(3-methyl-but-2-enyl)-1,4-dioxonaphthalen-2-yl]acetate $\}$ for the first time by Cooke et al. (1939). Jacobsen \& Torsell (1973) prepared lapachol acetate using 2-acetoxy-1,4naphthoquinone as a precursor with $79 \%$ yield. We developed an optimization of the first synthesis of lapachol acetate developed by Cooke et al. (1939), introducing several modifications with the purpose of standardizing it and increasing the yield to $97.5 \%$. Details of the synthesis and the spectroscopic characterization are included in the supporting information. Noting that the crystal structure of lapachol acetate had not been reported, we also undertook the crystallization and structure determination.

\section{Structural commentary}

The lapachol acetate molecule (Fig. 1) is the ester of lapachol at the alcohol moiety (O2 in Larsen et al., 1992). The molecule 
is composed of three planar groups, the napthoquinone nucleus comprising atoms $\mathrm{C} 1$ to $\mathrm{C} 11, \mathrm{O} 1, \mathrm{O} 2$ and $\mathrm{O} 4$, and two smaller butenyl and acetate residues at the sides. The butenyl and acetate mean planes are inclined to the naphthoquinone mean plane by $65.80(10)$ and $78.52(11)^{\circ}$, respectively. The lapachol acetate molecule shows typical bond distances and angles, and overlaps very closely with the common part of the lapachol molecule in the structure LAPA II reported by Larsen et al. (1992) (Fig. 2), with an average deviation of $\mathrm{C} / \mathrm{O}$ atomic positions of $0.158 \AA$ and a maximum deviation of $0.309 \AA$ for atom O4. This is rather unexpected, since the butenyl moiety shows rotational flexibility around the C3$\mathrm{C} 11$ and $\mathrm{C} 11-\mathrm{C} 12$ bonds. However, in both reported lapachol polymorphs (Larsen et al., 1992) and two derivatives (Eyong et al., 2015; da Silva et al., 2012) reported in the CSD (Groom et al., 2016) with the same rotational freedom, the dihedral angle between the planar $\mathrm{C}=\mathrm{C}\left(\mathrm{CH}_{3}\right)_{2}$ group and the naphthoquinone nucleus is close to $70^{\circ}$, as observed in lapachol acetate.
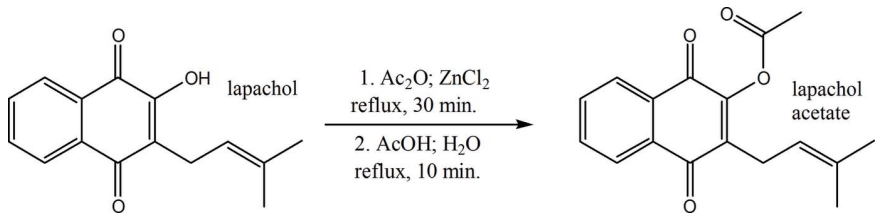

\section{Supramolecular features}

Crystals of lapachol acetate are held together by weak dipolar and dispersion forces because there is no strong $\mathrm{H}$-donor residue in the molecule. Molecules connect with other units through weak $\mathrm{C}\left(s p^{3}\right)-\mathrm{H} \cdots \mathrm{O}=\mathrm{C}$ hydrogen bonds $\mathrm{H} 11 B \cdots \mathrm{O} 4^{\mathrm{i}}$ and $\mathrm{H} 15 A \cdots \mathrm{O} 1^{\mathrm{ii}}$ (Table 1, Fig. S4a in the supporting information) defining double sheets of molecules parallel to (101). The butenyl residue of a screw-rotation-

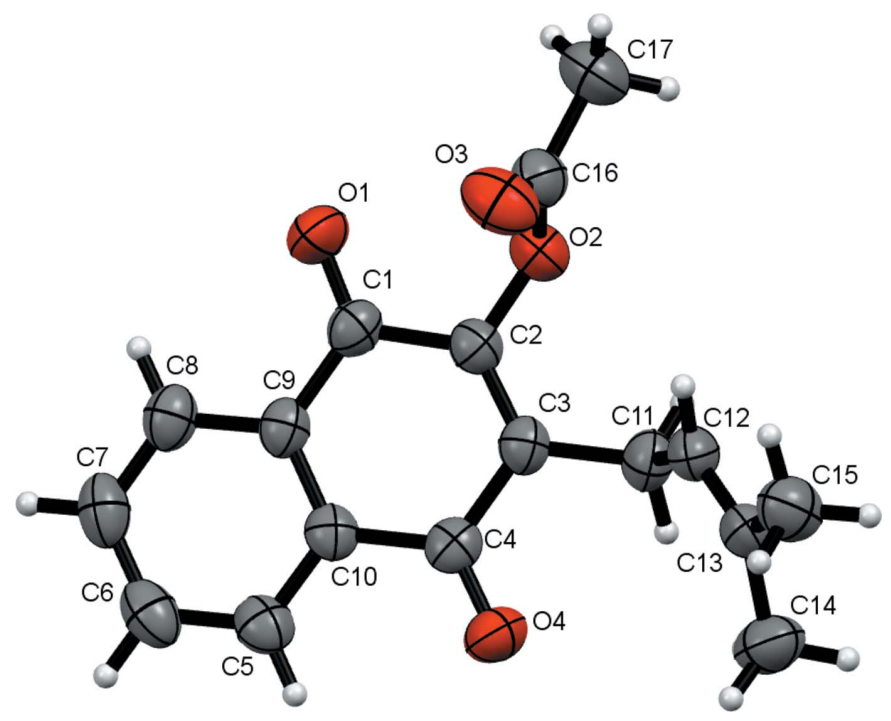

Figure 1

ORTEP view of a lapachol acetate molecule with the labelling scheme, and displacement ellipsoids drawn at the $50 \%$ probability level. One of the two positions of the disordered $\mathrm{C} 17$ methyl group has been omitted for clarity.
Table 1

Hydrogen-bond geometry $\left(\AA{ }^{\circ}\right)$.

\begin{tabular}{lllll}
\hline$D-\mathrm{H} \cdots A$ & $D-\mathrm{H}$ & $\mathrm{H} \cdots A$ & $D \cdots A$ & $D-\mathrm{H} \cdots A$ \\
\hline $\mathrm{C} 11-\mathrm{H} 11 B \cdots \mathrm{O} 4^{\mathrm{i}}$ & 0.97 & 2.55 & $3.274(3)$ & 131 \\
$\mathrm{C} 15-\mathrm{H} 15 A \cdots \mathrm{O} 1^{\mathrm{ii}}$ & 0.96 & 2.59 & $3.485(3)$ & 156 \\
\hline
\end{tabular}

Symmetry codes: (i) $-x+\frac{1}{2}, y-\frac{1}{2},-z+\frac{3}{2}$; (ii) $x+\frac{1}{2},-y+\frac{1}{2}, z+\frac{1}{2}$.

related molecule adds an intermolecular $\pi-\pi$ interaction with the naphtoquinone residue to the sheets with atoms $\mathrm{C} 12$ iii and $\mathrm{C} 13^{\mathrm{iii}}$ located at 3.243 and $3.544 \AA$, respectively, from the naphtoquinone plane (Fig. S4b). Finally, the double sheets stack along the [101] direction where naphthoquinone nuclei of inversion-related molecules display $\pi-\pi$ interactions. Ring 1 (C1-C4/C10/C9; centroid $C g 1$ ) of the molecule is close to ring 2 (C5-C10; centroid $C g 2$ ), the $C g 2 \cdots C g 1^{\text {iv }}$ distance being $3.8532(12) \AA) ; \quad C g 2 \cdots C g 2^{\text {iv }}$ is the shortest distance [3.8035(13) $\AA$ ] , with an average perpendicular distance between naphtoquinone planes of 3.3787 (9) $\AA$, as shown in Fig. S $4 c$ [symmetry codes: (i) $-x+\frac{1}{2}, y-\frac{1}{2},-z+\frac{3}{2}$; (ii) $x+\frac{1}{2}$, $-y+\frac{1}{2}, z+\frac{1}{2}$; (iii) $\frac{1}{2}-x, \frac{1}{2}+y, \frac{3}{2}-z$; (iv) $1-x, 1-y, 1-z$ ). Considering the Hirshfeld (HF) surface (Turner et al., 2017) mapped over $d_{\text {norm }}$ (analysis of the contact distances $d_{\mathrm{i}}$ and $d_{\mathrm{e}}$ from the HF surface to the nearest atom inside and outside, respectively), these interactions in one molecule are shown in Fig. $3 a$ and the sheets of molecules defined by them in Fig. $3 b$. The 2D fingerprints of lapachol acetate (shown in Fig. S5 of the supporting information) show no particular features other than the aforementioned $\mathrm{H} \cdots \mathrm{O} / \mathrm{O} \cdots \mathrm{H}$ contacts, which comprise $28.2 \%$ of the total HF surface, revealing their importance in the formation of the crystal.

In order to describe these interactions in a whole-of-molecule approach, accurate model energies of the interactions between molecules of lapachol acetate in the crystal were analysed. The interactions were calculated using the B3LYP/ 6-31 G(d,p) energy model implemented in CrystalExplorer (Turner et al., 2017), which uses quantum mechanical charge distributions for unperturbed molecules (Mackenzie et al., 2017). In the calculations, the total energy is modelled as the

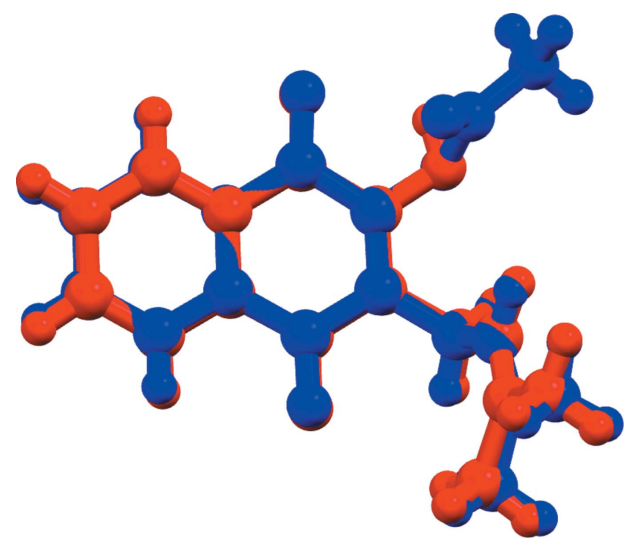

Figure 2

Overlay of a lapachol acetate (blue) and a lapachol (red) molecule (LAPA II molecule as described by Larsen et al., 1992). Only the common $\mathrm{C} / \mathrm{O}$ atoms were fitted. 


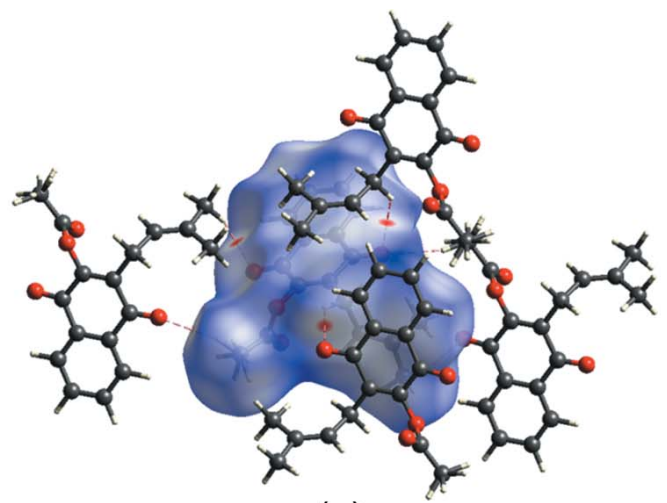

(a)

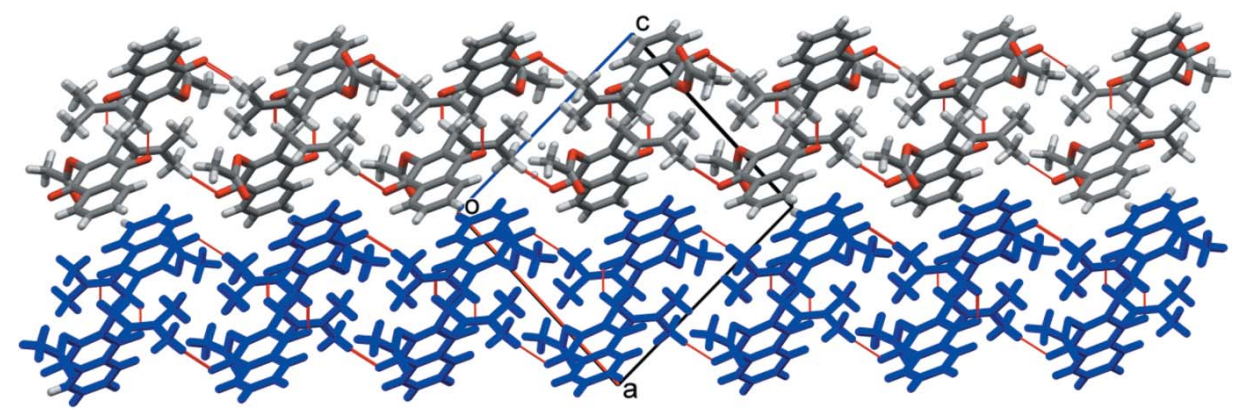

(b)

Figure 3

(a) View of the Hirshfeld surface for lapachol acetate mapped over $d_{\text {norm }}$ showing the $\mathrm{C}-\mathrm{H} \cdots \mathrm{O}$ hydrogen-bond interactions. $(b)$ The molecular structure of lapachol acetate showing the formation of stacked (101) sheets.

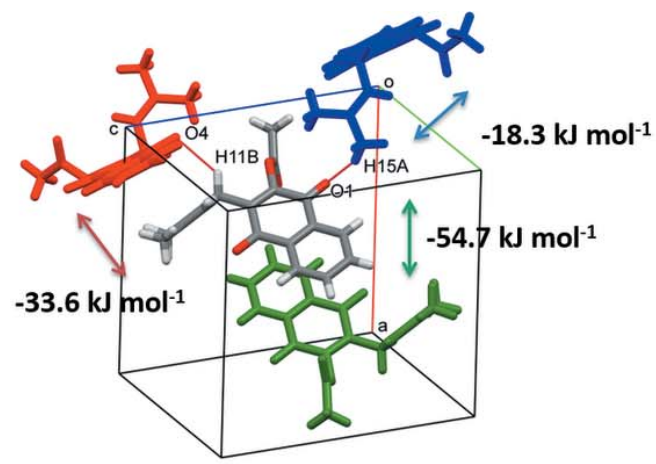

(a)

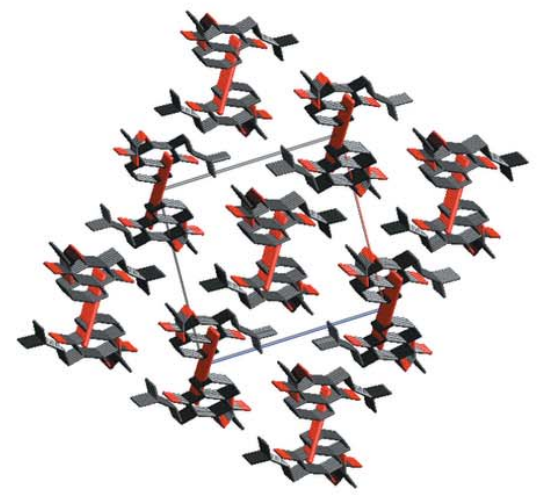

Figure 4

(a) Molecular close contacts and (b) energy-framework diagrams for electrostatic (red) and dispersion (green) contributions to the total interaction energy (blue) in lapachol acetate crystals.

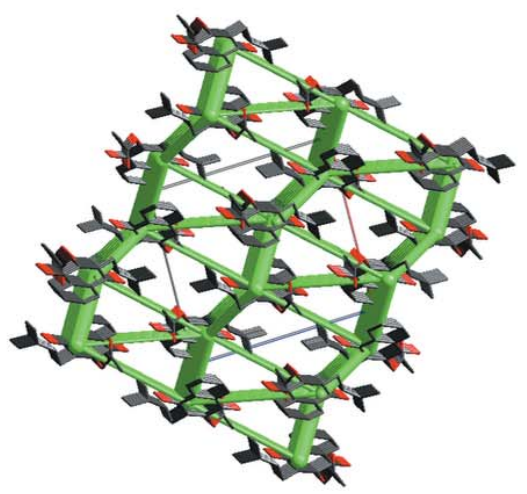

(b)

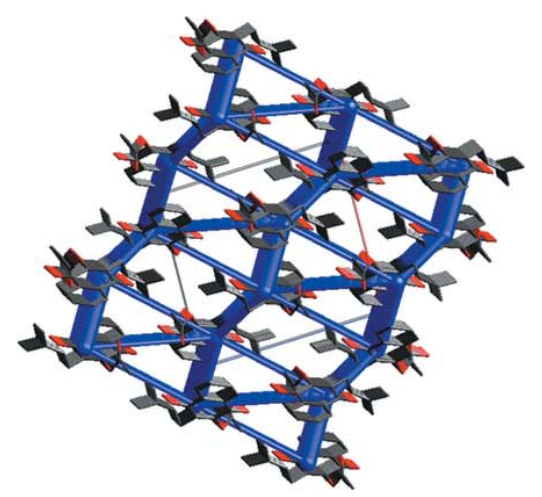


sum of the electrostatic $\left(E_{\text {ele }}\right)$, polarization $\left(E_{\mathrm{pol}}\right)$, dispersion $\left(E_{\mathrm{dis}}\right)$ and exchange-repulsion $\left(E_{\mathrm{rep}}\right)$ terms (Mackenzie et al., 2017). The strongest pairwise interaction with a total energy of $-54.7 \mathrm{~kJ} \mathrm{~mol}^{-1}$ corresponds to the interaction between neighbouring aromatic systems, while the molecules connected through a combination of $\pi-\pi$ interactions between the butenyl and naphtoquinone residues and $\mathrm{C} 11-$ $\mathrm{H} 11 B \cdots \mathrm{O} 4^{\mathrm{i}}$ hydrogen bonds have a total energy of $-33.6 \mathrm{~kJ} \mathrm{~mol}^{-1}$. The weakest interaction, $\mathrm{C} 15-\mathrm{H} 15 A \cdots \mathrm{O} 1^{\mathrm{ii}}$, shows a total energy of $-18.3 \mathrm{~kJ} \mathrm{~mol}^{-1}$ (Fig. $4 a$ ). The energy framework diagrams for lapachol acetate (Fig. 4b) show that electrostatic forces act to keep pairs of inversion-related chains of molecules joined while dispersion forces act in three dimensions to build the crystal structure. The total energy diagram shows a high resemblance to the dispersion framework, showing that these forces are the most important in the crystal. The interaction energies for selected molecular pairs in the first coordination sphere around the asymmetric unit are summarized in Table S1 and Fig. S6 of the supporting information.

\section{Database survey}

Lapachol and its derivatives are rather scarce in the Cambridge Crystal Structure Database (Version 5.40, update 2 of May 2019; Groom et al., 2016) with only 31 entries matching the basic $\mathrm{C}-\mathrm{O}$ framework of lapachol. Two lapachol polymorphs LAPA I and LAPA II (Larsen et al., 1992) have been reported at $105 \mathrm{~K}$, as mentioned above. Two additional lapachol derivatives obtained by replacing one $\mathrm{H}$ atom have been reported during the current decade: 4-(3-hydroxy-1,4-dioxo1,4-dihydronaphthalen-2-yl)-2-methylbut-2-enal (Eyong et al., 2015) is an aldehyde of lapachol at C15 and 3-(3-methylbut-2en-1-yl)-1,4-dioxo-1,4-dihydronaphthalen-2-yl 4-methylbenzenesulfonate (Silva et al., 2012) is a sulfonate at O2. Lapachol acetate is the third derivative of this kind reported. Some lapachol derivatives where cyclization of the 3-methy-2butenyl moiety or coordination with metals (as lapacholate) have additionally been reported, but are not related to lapachol acetate.

\section{Synthesis and crystallization}

Lapachol was obtained from an extract of Handroanthus Heptaphyllus (Vell.) Mattos, the pink trumpet tree (or lapacho negro) as described in the supporting information. $201 \mathrm{mg}$ $(0.823 \mathrm{mmol})$ of lapachol were dissolved in $5 \mathrm{ml}$ of dry acetic anhydride and a catalytic amount of dry zinc chloride $\left(\mathrm{ZnCl}_{2}\right)$ was added. The suspension was refluxed for $30 \mathrm{~min}$ under an inert atmosphere $\left(\mathrm{N}_{2}\right)$. The solution was allowed to cool and $5 \mathrm{ml}$ of glacial acetic acid and later $50 \mathrm{ml}$ of distilled water were added. The final mixture was refluxed for $10 \mathrm{~min}$ and allowed to precipitate overnight. The crude solid was filtered, dried and purified by column chromatography (hexane: AcOEt, 9: $1 v / v$ ) to obtain pure lapachol acetate as yellow crystals (see the detailed description of the obtention of lapachol and the synthesis of lapachol acetate in the
Table 2

Experimental details.

\begin{tabular}{ll}
\hline Crystal data & \\
Chemical formula & $\mathrm{C}_{17} \mathrm{H}_{16} \mathrm{O}_{4}$ \\
$M_{\mathrm{r}}$ & 284.30 \\
Crystal system, space group & Monoclinic, $P 2_{1} / n$ \\
Temperature $(\mathrm{K})$ & 296 \\
$a, b, c(\AA)$ & $12.0914(8), 9.4741(6), 12.7761(9)$ \\
$\beta\left({ }^{\circ}\right.$ & $92.943(4)$ \\
$V\left(\AA^{3}\right)$ & $1461.64(17)$ \\
$Z$ & 4 \\
Radiation type & $\mathrm{Cu} K \alpha$ \\
$\mu\left(\mathrm{mm}^{-1}\right)$ & 0.75 \\
Crystal size $(\mathrm{mm})$ & $0.26 \times 0.22 \times 0.18$ \\
& \\
Data collection & $\mathrm{Bruker} \mathrm{D} 8 \mathrm{Venture} /$ Photon 100 \\
Diffractometer & $\mathrm{CMOS}$ \\
& Multi-scan $(S A D A B S ;$ Krause $e t$ \\
Absorption correction & al. 2015$)$ \\
& $0.654,0.754$ \\
$T_{\min }, T_{\max }$ & $7496,2996,1972$ \\
No. of measured, independent and & \\
$\quad$ observed $[I>2 \sigma(I)]$ reflections & 0.038 \\
$R_{\text {int }}$ & 0.637 \\
$(\text { sin } \theta / \lambda)_{\text {max }}\left(\AA^{-1}\right)$ & \\
Refinement & \\
$R\left[F^{2}>2 \sigma\left(F^{2}\right)\right], w R\left(F^{2}\right), S$ & $0.048,0.136,1.03$ \\
No. of reflections & 2996 \\
No. of parameters & 193 \\
$\mathrm{H}$-atom treatment & $\mathrm{H}$-atom parameters constrained \\
$\Delta \rho_{\text {max }}, \Delta \rho_{\text {min }}\left(\mathrm{e} \AA^{-3}\right)$ & $0.17,-0.16$ \\
\hline
\end{tabular}

Computer programs: APEX2 and SAINT (Bruker, 2014), SHELXT (Sheldrick, 2015a), SHELXL2018 (Sheldrick, 2015b), Mercury (Macrae et al., 2008) and publCIF (Westrip, 2010)

supporting information and the detailed spectroscopic study in Figs. S1-S3). Adequate crystals for diffraction were obtained by dissolving a few $\mathrm{mg}$ of the solid in a minimum amount of AcOEt in a rubber-stop vial with a syringe needle through the center to promote slow evaporation of the solvent at room temperature.

\section{Refinement}

Crystal data, data collection and structure refinement details are summarized in Table 2. $\mathrm{H}$ atoms were placed in calculated positions $(\mathrm{C}-\mathrm{H}=0.93-0.97 \AA)$ and included as riding contributions, with isotropic displacement parameters set at 1.2-1.5 times the $U_{\text {eq }}$ value of the parent atom. The C17 methyl group shows rotational disorder that was modelled with two positions that were refined with a fixed $\mathrm{C}-\mathrm{H}$ bond distance but with rotational freedom (AFIX 147) converging at occupancies of 0.79 (3) and 0.21 (3).

\section{Acknowledgements}

The authors are indebted to N. Di Benedetto for her contribution to the single-crystal X-ray diffraction data processing and to G. Cebrián-Torrejón for his assistance in the spectroscopic characterization. Funding for this research was provided by Programa de Desarrollo de Ciencias Basicas PEDECIBA - Uruguay (grant to Leopoldo Suescun, Enrique 
Pandolfi); Facultad de Ciencias Exactas y Naturales, Universidad Nacional de Asunción (Paraguay).

\section{References}

Araújo, I. A. C., de Paula, R. C., Alves, C. L., Faria, K. F., Oliveira, M. M., Mendes, G. G., Dias, E. M. F. A., Ribeiro, R. R., Oliveira, A. B. \& Silva, S. M. D. (2019). Exp. Parasitol. 199, 67-73.

Babula, P., Adam, V., Havel, L. \& Kizek, R. (2007). Ceska Slov Farm Cas Ceske Farm Spolecnosti Slov Farm Spolecnosti, 56, 114-20.

Barbosa Coitinho, L., Fumagalli, F., da Rosa-Garzon, N. G., da Silva Emery, F. \& Cabral, H. (2019). Prep. Biochem. Biotechnol. 49, 459463.

Bruker (2014). APEX2, SAINT and SADABS. Bruker AXS Inc., Madison, Wisconsin, USA.

Bruneton, J. (2001). Farmacognosia. Fitoquímica. Plantas medicinales, edited by A. Villar del Fresno, E. Carretero and M. Rebuelta. Spain: ACRIBIA Editorial S. A.

Cooke, R. G., Macbeth, A. K. \& Winzor, F. L. (1939). J. Chem. Soc. pp. 878-884.

Eyong, K. O., Ketsemen, H. L., Ghansenyuy, S. Y. \& Folefoc, G. N. (2015). Med. Chem. Res. 24, 965-969.

Groom, C. R., Bruno, I. J., Lightfoot, M. P. \& Ward, S. C. (2016). Acta Cryst. B72, 171-179.

Jacobsen, N. \& Torsell, K. (1973). Acta Chem. Scand. 27, 32113216.

Krause, L., Herbst-Irmer, R., Sheldrick, G. M. \& Stalke, D. (2015). J. Appl. Cryst. 48, 3-10.
Larsen, I. K., Andersen, L. A. \& Pedersen, B. F. (1992). Acta Cryst. C48, 2009-2013.

Mackenzie, C. F., Spackman, P. R., Jayatilaka, D. \& Spackman, M. A. (2017). IUCrJ, 4, 575-587.

Macrae, C. F., Bruno, I. J., Chisholm, J. A., Edgington, P. R., McCabe, P., Pidcock, E., Rodriguez-Monge, L., Taylor, R., van de Streek, J. \& Wood, P. A. (2008). J. Appl. Cryst. 41, 466-470.

Miranda, S. E., Lemos, J. A., Fernandes, R. S., Ottoni, F. M., Alves, R. J., Ferretti, A., Rubello, D., Cardoso, V. N. \& Branco de Barrosa, A. L. (2019). Rev. Esp. Med. Nucl. Ima. 38, 167-172.

Sheldrick, G. M. (2015a). Acta Cryst. A71, 3-8.

Sheldrick, G. M. (2015b). Acta Cryst. C71, 3-8.

Silva, E. N. da de Júnior, Melo, I. M. M., Diogo, E. B. T., Costa, V. A., de Souza Filho, J. D., Valença, W. O., Camara, C. A., de Oliveira, R. N., de Araujo, A. S., Emery, F. S., dos Santos, M. R., de Simone, C. A., Menna-Barreto, R. F. S. \& de Castro, S. L. (2012). Eur. J. Med. Chem. 52, 304-312.

Silva, F. C. da \& Ferreira, V. F. (2016). Curr. Org. Synth. 13, 334-371. Strauch, M. A., Tomaz, M. A., Monteiro-Machado, M., Cons, B. L., Patrão-Neto, F. C., Teixeira-Cruz, J. D. M., Tavares-Henriques, M. D. S., Nogueira-Souza, P. D., Gomes, S. L. S., Costa, P. R. R., Schaeffer, E., da Silva, A. J. M. \& Melo, P. A. (2019). PLoS One, 14, e0211229.

Turner, M. J., McKinnon, J. J., Wolff, S. K., Grimwood, D. J., Spackman, P. R., Jayatilaka, D. \& Spackman, M. A. (2017). CrystalExplorer17. University of Western Australia. http://hirshfeldsurface.net.

Westrip, S. P. (2010). J. Appl. Cryst. 43, 920-925. 


\section{supporting information}

Acta Cryst. (2019). E75, 1362-1366 [https://doi.org/10.1107/S2056989019011393]

\section{Crystal structure and Hirshfeld surface analysis of lapachol acetate $\mathbf{8 0}$ years}

\section{after its first synthesis}

Miguel A. Martínez-Cabrera, Mario A. Macías, Francisco Ferreira, Enrique Pandolfi, Javier

\section{Barúa and Leopoldo Suescun}

\section{Computing details}

Data collection: APEX2 (Bruker, 2014); cell refinement: SAINT (Bruker, 2014); data reduction: SAINT (Bruker, 2014); program(s) used to solve structure: SHELXT (Sheldrick, 2015a); program(s) used to refine structure: SHELXL2018 (Sheldrick, 2015b); molecular graphics: Mercury (Macrae et al., 2008); software used to prepare material for publication: publCIF (Westrip, 2010).

3-(3-Methylbut-2-enyl)-1,4-dioxonaphthalen-2-yl acetate

Crystal data

$\mathrm{C}_{17} \mathrm{H}_{16} \mathrm{O}_{4}$

$M_{r}=284.30$

Monoclinic, $P 2{ }_{1} / n$

$a=12.0914(8) \AA$

$b=9.4741(6) \AA$

$c=12.7761(9) \AA$

$\beta=92.943(4)^{\circ}$

$V=1461.64(17) \AA^{3}$

$Z=4$

$F(000)=600$

\section{Data collection}

Bruker D8 Venture/Photon 100 CMOS diffractometer

Radiation source: $\mathrm{Cu}$ Incoatec microsource

Detector resolution: 10.4167 pixels $\mathrm{mm}^{-1}$

$\mathrm{j}$ and $\omega$ scans

Absorption correction: multi-scan

(SADABS; Krause et al., 2015)

$T_{\min }=0.654, T_{\max }=0.754$

\section{Refinement}

Refinement on $F^{2}$

Least-squares matrix: full

$R\left[F^{2}>2 \sigma\left(F^{2}\right)\right]=0.048$

$w R\left(F^{2}\right)=0.136$

$S=1.03$

2996 reflections

193 parameters

0 restraints
$D_{\mathrm{x}}=1.292 \mathrm{Mg} \mathrm{m}^{-3}$

Melting point: 352(1) $\mathrm{K}$

$\mathrm{Cu} K \alpha$ radiation, $\lambda=1.54178 \AA$

Cell parameters from 7958 reflections

$\theta=4.9-75.7^{\circ}$

$\mu=0.75 \mathrm{~mm}^{-1}$

$T=296 \mathrm{~K}$

Block, yellow

$0.26 \times 0.22 \times 0.18 \mathrm{~mm}$

7496 measured reflections

2996 independent reflections

1972 reflections with $I>2 \sigma(I)$

$R_{\text {int }}=0.038$

$\theta_{\max }=79.2^{\circ}, \theta_{\min }=4.9^{\circ}$

$h=-13 \rightarrow 15$

$k=-11 \rightarrow 9$

$l=-16 \rightarrow 15$

Primary atom site location: dual

Secondary atom site location: difference Fourier map

Hydrogen site location: inferred from neighbouring sites

$\mathrm{H}$-atom parameters constrained

$w=1 /\left[\sigma^{2}\left(F_{\mathrm{o}}{ }^{2}\right)+(0.053 P)^{2}+0.3463 P\right]$

where $P=\left(F_{\mathrm{o}}^{2}+2 F_{\mathrm{c}}{ }^{2}\right) / 3$ 
$(\Delta / \sigma)_{\max }<0.001$

$\Delta \rho_{\max }=0.17$ e $\AA^{-3}$

Special details

Geometry. All esds (except the esd in the dihedral angle between two 1.s. planes) are estimated using the full covariance matrix. The cell esds are taken into account individually in the estimation of esds in distances, angles and torsion angles; correlations between esds in cell parameters are only used when they are defined by crystal symmetry. An approximate (isotropic) treatment of cell esds is used for estimating esds involving l.s. planes.

Fractional atomic coordinates and isotropic or equivalent isotropic displacement parameters $\left(\AA^{2}\right)$

\begin{tabular}{|c|c|c|c|c|c|}
\hline & $x$ & $y$ & $z$ & $U_{\text {iso }} * / U_{\text {eq }}$ & Occ. $(<1)$ \\
\hline $\mathrm{O} 1$ & $0.22182(14)$ & $0.34297(18)$ & $0.34233(12)$ & $0.0686(5)$ & \\
\hline $\mathrm{C} 1$ & $0.25877(15)$ & $0.3726(2)$ & $0.42998(15)$ & $0.0468(5)$ & \\
\hline $\mathrm{O} 2$ & $0.17186(11)$ & $0.16221(16)$ & $0.49500(11)$ & $0.0540(4)$ & \\
\hline $\mathrm{C} 2$ & $0.24117(15)$ & $0.2761(2)$ & $0.51890(15)$ & $0.0441(5)$ & \\
\hline $\mathrm{O} 3$ & $0.30987(14)$ & $0.05735(18)$ & $0.41410(15)$ & $0.0756(5)$ & \\
\hline $\mathrm{C} 3$ & $0.28099(15)$ & $0.2973(2)$ & $0.61720(15)$ & $0.0443(5)$ & \\
\hline $\mathrm{O} 4$ & $0.39859(12)$ & $0.43627(17)$ & $0.72673(11)$ & $0.0586(4)$ & \\
\hline $\mathrm{C} 4$ & $0.35332(15)$ & $0.4218(2)$ & $0.64003(14)$ & $0.0440(5)$ & \\
\hline $\mathrm{C} 5$ & $0.42753(17)$ & $0.6488(2)$ & $0.57604(17)$ & $0.0529(5)$ & \\
\hline H5 & 0.460252 & 0.663841 & 0.642578 & $0.063 *$ & \\
\hline C6 & $0.43917(18)$ & $0.7485(3)$ & $0.49821(19)$ & $0.0615(6)$ & \\
\hline H6 & 0.479948 & 0.830043 & 0.512619 & $0.074 *$ & \\
\hline $\mathrm{C} 7$ & $0.39054(19)$ & $0.7275(3)$ & $0.39920(19)$ & $0.0612(6)$ & \\
\hline $\mathrm{H} 7$ & 0.397581 & 0.795506 & 0.347504 & $0.073 *$ & \\
\hline $\mathrm{C} 8$ & $0.33163(17)$ & $0.6057(3)$ & 0.37709 (17) & $0.0547(5)$ & \\
\hline $\mathrm{H} 8$ & 0.299507 & 0.591249 & 0.310242 & $0.066^{*}$ & \\
\hline C9 & $0.32006(15)$ & $0.5041(2)$ & $0.45465(15)$ & $0.0444(5)$ & \\
\hline $\mathrm{C} 10$ & $0.36737(15)$ & $0.5268(2)$ & $0.55524(15)$ & $0.0426(4)$ & \\
\hline C11 & $0.26046(18)$ & $0.1969(2)$ & $0.70541(16)$ & $0.0533(5)$ & \\
\hline H11A & 0.260239 & 0.249275 & 0.770631 & $0.064 *$ & \\
\hline H11B & 0.188017 & 0.154157 & 0.693426 & $0.064 *$ & \\
\hline $\mathrm{C} 12$ & $0.34630(17)$ & $0.0828(2)$ & $0.71541(16)$ & $0.0505(5)$ & \\
\hline H12 & 0.350981 & 0.023269 & 0.657923 & $0.061^{*}$ & \\
\hline $\mathrm{C} 13$ & $0.41622(17)$ & $0.0566(2)$ & $0.79619(16)$ & $0.0510(5)$ & \\
\hline C14 & $0.4219(2)$ & $0.1431(3)$ & 0.89505 (18) & $0.0719(7)$ & \\
\hline H14A & 0.412225 & 0.082669 & 0.954178 & $0.108^{*}$ & \\
\hline H14B & 0.364369 & 0.213071 & 0.891358 & $0.108 *$ & \\
\hline $\mathrm{H} 14 \mathrm{C}$ & 0.492685 & 0.188880 & 0.902682 & $0.108^{*}$ & \\
\hline $\mathrm{C} 15$ & $0.4952(2)$ & $-0.0652(3)$ & $0.7946(2)$ & $0.0684(7)$ & \\
\hline H15A & 0.569682 & -0.031304 & 0.805937 & $0.103 *$ & \\
\hline H15B & 0.487694 & -0.111606 & 0.727755 & $0.103 *$ & \\
\hline $\mathrm{H} 15 \mathrm{C}$ & 0.478890 & -0.130827 & 0.848916 & $0.103 *$ & \\
\hline $\mathrm{C} 16$ & $0.2148(2)$ & $0.0591(2)$ & $0.43364(17)$ & $0.0557(5)$ & \\
\hline $\mathrm{C} 17$ & $0.1276(2)$ & $-0.0443(3)$ & $0.3994(2)$ & $0.0785(8)$ & $0.79(3)$ \\
\hline H17A & 0.072901 & 0.001798 & 0.354279 & $0.118 *$ & $0.79(3)$ \\
\hline H17B & 0.093164 & -0.081079 & 0.459726 & $0.118^{*}$ & $0.79(3)$ \\
\hline
\end{tabular}




$\begin{array}{llllll}\text { H17C } & 0.160520 & -0.120203 & 0.362194 & 0.118^{*} & 0.79(3) \\ \text { C17' } & 0.1276(2) & -0.0443(3) & 0.3994(2) & 0.0785(8) & 0.21(3) \\ \text { H17D } & 0.058095 & -0.016088 & 0.425921 & 0.118^{*} & 0.21(3) \\ \text { H17E } & 0.147328 & -0.136138 & 0.426025 & 0.118^{*} & 0.21(3) \\ \text { H17F } & 0.121161 & -0.047259 & 0.324252 & 0.118^{*} & 0.21(3)\end{array}$

Atomic displacement parameters $\left(\AA^{2}\right)$

\begin{tabular}{|c|c|c|c|c|c|c|}
\hline & $U^{11}$ & $U^{22}$ & $U^{33}$ & $U^{12}$ & $U^{13}$ & $U^{23}$ \\
\hline O1 & 0.0903 (12) & $0.0652(12)$ & $0.0484(8)$ & $-0.0047(9)$ & $-0.0162(8)$ & $0.0054(8)$ \\
\hline $\mathrm{C} 1$ & $0.0471(10)$ & $0.0480(12)$ & $0.0449(11)$ & $0.0085(9)$ & $-0.0009(8)$ & $0.0029(9)$ \\
\hline $\mathrm{O} 2$ & $0.0536(8)$ & $0.0488(9)$ & $0.0596(8)$ & $-0.0054(7)$ & $0.0025(7)$ & $-0.0014(7)$ \\
\hline $\mathrm{C} 2$ & $0.0430(9)$ & 0.0407 (11) & $0.0486(10)$ & $0.0036(9)$ & $0.0025(8)$ & $0.0002(9)$ \\
\hline $\mathrm{O} 3$ & $0.0664(10)$ & $0.0607(12)$ & 0.1004 (13) & $0.0026(9)$ & $0.0100(9)$ & $-0.0191(10)$ \\
\hline $\mathrm{C} 3$ & $0.0449(10)$ & $0.0430(12)$ & $0.0456(10)$ & $0.0058(9)$ & $0.0060(8)$ & $0.0037(9)$ \\
\hline $\mathrm{O} 4$ & $0.0653(9)$ & $0.0646(11)$ & $0.0452(8)$ & $0.0030(8)$ & $-0.0055(7)$ & $-0.0027(7)$ \\
\hline $\mathrm{C} 4$ & $0.0431(10)$ & $0.0448(12)$ & $0.0443(10)$ & $0.0105(9)$ & $0.0051(8)$ & $-0.0028(9)$ \\
\hline $\mathrm{C} 5$ & $0.0557(12)$ & $0.0456(13)$ & $0.0581(12)$ & $0.0034(10)$ & $0.0095(10)$ & $-0.0063(11)$ \\
\hline C6 & $0.0621(13)$ & $0.0424(14)$ & $0.0813(16)$ & $-0.0016(11)$ & $0.0171(12)$ & $-0.0029(12)$ \\
\hline $\mathrm{C} 7$ & $0.0673(14)$ & $0.0464(14)$ & $0.0715(15)$ & $0.0066(11)$ & $0.0198(12)$ & $0.0138(12)$ \\
\hline $\mathrm{C} 8$ & $0.0571(12)$ & $0.0534(14)$ & $0.0539(12)$ & $0.0109(11)$ & $0.0061(9)$ & $0.0098(11)$ \\
\hline C9 & $0.0457(10)$ & $0.0428(12)$ & $0.0452(10)$ & $0.0088(9)$ & $0.0065(8)$ & 0.0049 (9) \\
\hline $\mathrm{C} 10$ & $0.0431(9)$ & $0.0373(11)$ & $0.0479(10)$ & $0.0086(8)$ & $0.0078(8)$ & $-0.0009(9)$ \\
\hline $\mathrm{C} 11$ & $0.0566(12)$ & $0.0559(14)$ & $0.0479(11)$ & $-0.0022(10)$ & 0.0067 (9) & $0.0092(10)$ \\
\hline $\mathrm{C} 12$ & $0.0625(12)$ & $0.0431(13)$ & $0.0458(10)$ & $-0.0042(10)$ & $0.0010(9)$ & $0.0028(9)$ \\
\hline $\mathrm{C} 13$ & $0.0543(11)$ & $0.0490(13)$ & $0.0494(11)$ & $-0.0102(10)$ & $-0.0005(9)$ & $0.0040(10)$ \\
\hline $\mathrm{C} 14$ & $0.0739(15)$ & $0.086(2)$ & 0.0545 (13) & $-0.0051(14)$ & $-0.0078(11)$ & $-0.0076(13)$ \\
\hline $\mathrm{C} 15$ & $0.0697(14)$ & $0.0583(16)$ & $0.0756(15)$ & 0.0019 (13) & $-0.0109(12)$ & $0.0043(13)$ \\
\hline $\mathrm{C} 16$ & $0.0658(13)$ & 0.0453 (13) & $0.0556(12)$ & $-0.0005(11)$ & $-0.0019(10)$ & $0.0025(10)$ \\
\hline $\mathrm{C} 17$ & $0.0874(18)$ & $0.0623(18)$ & $0.0851(18)$ & $-0.0195(14)$ & $-0.0028(14)$ & $-0.0071(15)$ \\
\hline $\mathrm{C} 17^{\prime}$ & $0.0874(18)$ & $0.0623(18)$ & $0.0851(18)$ & $-0.0195(14)$ & $-0.0028(14)$ & $-0.0071(15)$ \\
\hline
\end{tabular}

Geometric parameters $\left(A,{ }^{\circ}\right)$

\begin{tabular}{llll}
\hline $\mathrm{O} 1-\mathrm{C} 1$ & $1.217(2)$ & $\mathrm{C} 11-\mathrm{C} 12$ & $1.500(3)$ \\
$\mathrm{C} 1-\mathrm{C} 9$ & $1.476(3)$ & $\mathrm{C} 11-\mathrm{H} 11 \mathrm{~A}$ & 0.9700 \\
$\mathrm{C} 1-\mathrm{C} 2$ & $1.482(3)$ & $\mathrm{C} 11-\mathrm{H} 11 \mathrm{~B}$ & 0.9700 \\
$\mathrm{O} 2-\mathrm{C} 16$ & $1.371(3)$ & $\mathrm{C} 12-\mathrm{C} 13$ & $1.324(3)$ \\
$\mathrm{O} 2-\mathrm{C} 2$ & $1.391(2)$ & $\mathrm{C} 12-\mathrm{H} 12$ & 0.9300 \\
$\mathrm{C} 2-\mathrm{C} 3$ & $1.337(3)$ & $\mathrm{C} 13-\mathrm{C} 15$ & $1.499(3)$ \\
$\mathrm{O} 3-\mathrm{C} 16$ & $1.189(3)$ & $\mathrm{C} 13-\mathrm{C} 14$ & $1.504(3)$ \\
$\mathrm{C} 3-\mathrm{C} 4$ & $1.489(3)$ & $\mathrm{C} 14-\mathrm{H} 14 \mathrm{~A}$ & 0.9600 \\
$\mathrm{C} 3-\mathrm{C} 11$ & $1.505(3)$ & $\mathrm{C} 14-\mathrm{H} 14 \mathrm{~B}$ & 0.9600 \\
$\mathrm{O} 4-\mathrm{C} 4$ & $1.218(2)$ & $\mathrm{C} 14-\mathrm{H} 14 \mathrm{C}$ & 0.9600 \\
$\mathrm{C} 4-\mathrm{C} 10$ & $1.487(3)$ & $\mathrm{C} 15-\mathrm{H} 15 \mathrm{~A}$ & 0.9600 \\
$\mathrm{C} 5-\mathrm{C} 6$ & $1.384(3)$ & $\mathrm{C} 15-\mathrm{H} 15 \mathrm{~B}$ & 0.9600 \\
$\mathrm{C} 5-\mathrm{C} 10$ & $1.384(3)$ & $\mathrm{C} 15-\mathrm{H} 15 \mathrm{C}$ & 0.9600 \\
$\mathrm{C} 5-\mathrm{H} 5$ & 0.9300 & $\mathrm{C} 16-\mathrm{C} 17^{\prime}$ & $1.488(3)$
\end{tabular}




\begin{tabular}{|c|c|c|c|}
\hline $\mathrm{C} 6-\mathrm{C} 7$ & $1.382(3)$ & $\mathrm{C} 16-\mathrm{C} 17$ & $1.488(3)$ \\
\hline $\mathrm{C} 6-\mathrm{H} 6$ & 0.9300 & $\mathrm{C} 17-\mathrm{H} 17 \mathrm{~A}$ & 0.9600 \\
\hline $\mathrm{C} 7-\mathrm{C} 8$ & $1.377(3)$ & C17-H17B & 0.9600 \\
\hline $\mathrm{C} 7-\mathrm{H} 7$ & 0.9300 & $\mathrm{C} 17-\mathrm{H} 17 \mathrm{C}$ & 0.9600 \\
\hline $\mathrm{C} 8-\mathrm{C} 9$ & $1.394(3)$ & $\mathrm{C} 17^{\prime}-\mathrm{H} 17 \mathrm{D}$ & 0.9600 \\
\hline $\mathrm{C} 8-\mathrm{H} 8$ & 0.9300 & $\mathrm{C} 17^{\prime}-\mathrm{H} 17 \mathrm{E}$ & 0.9600 \\
\hline $\mathrm{C} 9-\mathrm{C} 10$ & $1.396(3)$ & $\mathrm{C} 17^{\prime}-\mathrm{H} 17 \mathrm{~F}$ & 0.9600 \\
\hline $\mathrm{O} 1-\mathrm{C} 1-\mathrm{C} 9$ & $123.21(19)$ & $\mathrm{H} 11 \mathrm{~A}-\mathrm{C} 11-\mathrm{H} 11 \mathrm{~B}$ & 107.9 \\
\hline $\mathrm{O} 1-\mathrm{C} 1-\mathrm{C} 2$ & $120.2(2)$ & $\mathrm{C} 13-\mathrm{C} 12-\mathrm{C} 11$ & $127.8(2)$ \\
\hline $\mathrm{C} 9-\mathrm{C} 1-\mathrm{C} 2$ & $116.57(16)$ & $\mathrm{C} 13-\mathrm{C} 12-\mathrm{H} 12$ & 116.1 \\
\hline $\mathrm{C} 16-\mathrm{O} 2-\mathrm{C} 2$ & $115.91(16)$ & $\mathrm{C} 11-\mathrm{C} 12-\mathrm{H} 12$ & 116.1 \\
\hline $\mathrm{C} 3-\mathrm{C} 2-\mathrm{O} 2$ & $120.42(18)$ & $\mathrm{C} 12-\mathrm{C} 13-\mathrm{C} 15$ & $121.0(2)$ \\
\hline $\mathrm{C} 3-\mathrm{C} 2-\mathrm{C} 1$ & $124.68(19)$ & $\mathrm{C} 12-\mathrm{C} 13-\mathrm{C} 14$ & $123.5(2)$ \\
\hline $\mathrm{O} 2-\mathrm{C} 2-\mathrm{C} 1$ & $114.76(16)$ & $\mathrm{C} 15-\mathrm{C} 13-\mathrm{C} 14$ & $115.45(19)$ \\
\hline $\mathrm{C} 2-\mathrm{C} 3-\mathrm{C} 4$ & $118.84(18)$ & $\mathrm{C} 13-\mathrm{C} 14-\mathrm{H} 14 \mathrm{~A}$ & 109.5 \\
\hline $\mathrm{C} 2-\mathrm{C} 3-\mathrm{C} 11$ & $122.93(19)$ & $\mathrm{C} 13-\mathrm{C} 14-\mathrm{H} 14 \mathrm{~B}$ & 109.5 \\
\hline $\mathrm{C} 4-\mathrm{C} 3-\mathrm{C} 11$ & $118.16(17)$ & $\mathrm{H} 14 \mathrm{~A}-\mathrm{C} 14-\mathrm{H} 14 \mathrm{~B}$ & 109.5 \\
\hline $\mathrm{O} 4-\mathrm{C} 4-\mathrm{C} 10$ & $121.63(19)$ & $\mathrm{C} 13-\mathrm{C} 14-\mathrm{H} 14 \mathrm{C}$ & 109.5 \\
\hline $\mathrm{O} 4-\mathrm{C} 4-\mathrm{C} 3$ & $119.97(19)$ & $\mathrm{H} 14 \mathrm{~A}-\mathrm{C} 14-\mathrm{H} 14 \mathrm{C}$ & 109.5 \\
\hline $\mathrm{C} 10-\mathrm{C} 4-\mathrm{C} 3$ & $118.40(16)$ & $\mathrm{H} 14 \mathrm{~B}-\mathrm{C} 14-\mathrm{H} 14 \mathrm{C}$ & 109.5 \\
\hline $\mathrm{C} 6-\mathrm{C} 5-\mathrm{C} 10$ & $120.2(2)$ & $\mathrm{C} 13-\mathrm{C} 15-\mathrm{H} 15 \mathrm{~A}$ & 109.5 \\
\hline $\mathrm{C} 6-\mathrm{C} 5-\mathrm{H} 5$ & 119.9 & $\mathrm{C} 13-\mathrm{C} 15-\mathrm{H} 15 \mathrm{~B}$ & 109.5 \\
\hline $\mathrm{C} 10-\mathrm{C} 5-\mathrm{H} 5$ & 119.9 & $\mathrm{H} 15 \mathrm{~A}-\mathrm{C} 15-\mathrm{H} 15 \mathrm{~B}$ & 109.5 \\
\hline $\mathrm{C} 7-\mathrm{C} 6-\mathrm{C} 5$ & $120.4(2)$ & $\mathrm{C} 13-\mathrm{C} 15-\mathrm{H} 15 \mathrm{C}$ & 109.5 \\
\hline $\mathrm{C} 7-\mathrm{C} 6-\mathrm{H} 6$ & 119.8 & $\mathrm{H} 15 \mathrm{~A}-\mathrm{C} 15-\mathrm{H} 15 \mathrm{C}$ & 109.5 \\
\hline $\mathrm{C} 5-\mathrm{C} 6-\mathrm{H} 6$ & 119.8 & $\mathrm{H} 15 \mathrm{~B}-\mathrm{C} 15-\mathrm{H} 15 \mathrm{C}$ & 109.5 \\
\hline $\mathrm{C} 8-\mathrm{C} 7-\mathrm{C} 6$ & $120.0(2)$ & $\mathrm{O} 3-\mathrm{C} 16-\mathrm{O} 2$ & $121.9(2)$ \\
\hline $\mathrm{C} 8-\mathrm{C} 7-\mathrm{H} 7$ & 120.0 & $\mathrm{O} 3-\mathrm{C} 16-\mathrm{C} 17^{\prime}$ & $127.4(2)$ \\
\hline $\mathrm{C} 6-\mathrm{C} 7-\mathrm{H} 7$ & 120.0 & $\mathrm{O} 2-\mathrm{C} 16-\mathrm{C} 17^{\prime}$ & $110.7(2)$ \\
\hline $\mathrm{C} 7-\mathrm{C} 8-\mathrm{C} 9$ & $120.2(2)$ & $\mathrm{O} 3-\mathrm{C} 16-\mathrm{C} 17$ & $127.4(2)$ \\
\hline $\mathrm{C} 7-\mathrm{C} 8-\mathrm{H} 8$ & 119.9 & $\mathrm{O} 2-\mathrm{C} 16-\mathrm{C} 17$ & $110.7(2)$ \\
\hline $\mathrm{C} 9-\mathrm{C} 8-\mathrm{H} 8$ & 119.9 & $\mathrm{C} 16-\mathrm{C} 17-\mathrm{H} 17 \mathrm{~A}$ & 109.5 \\
\hline $\mathrm{C} 8-\mathrm{C} 9-\mathrm{C} 10$ & $119.8(2)$ & $\mathrm{C} 16-\mathrm{C} 17-\mathrm{H} 17 \mathrm{~B}$ & 109.5 \\
\hline $\mathrm{C} 8-\mathrm{C} 9-\mathrm{C} 1$ & $119.93(18)$ & $\mathrm{H} 17 \mathrm{~A}-\mathrm{C} 17-\mathrm{H} 17 \mathrm{~B}$ & 109.5 \\
\hline $\mathrm{C} 10-\mathrm{C} 9-\mathrm{C} 1$ & $120.30(18)$ & $\mathrm{C} 16-\mathrm{C} 17-\mathrm{H} 17 \mathrm{C}$ & 109.5 \\
\hline $\mathrm{C} 5-\mathrm{C} 10-\mathrm{C} 9$ & $119.48(19)$ & $\mathrm{H} 17 \mathrm{~A}-\mathrm{C} 17-\mathrm{H} 17 \mathrm{C}$ & 109.5 \\
\hline $\mathrm{C} 5-\mathrm{C} 10-\mathrm{C} 4$ & $119.83(18)$ & $\mathrm{H} 17 \mathrm{~B}-\mathrm{C} 17-\mathrm{H} 17 \mathrm{C}$ & 109.5 \\
\hline $\mathrm{C} 9-\mathrm{C} 10-\mathrm{C} 4$ & $120.69(19)$ & $\mathrm{C} 16-\mathrm{C} 17^{\prime}-\mathrm{H} 17 \mathrm{D}$ & 109.5 \\
\hline $\mathrm{C} 12-\mathrm{C} 11-\mathrm{C} 3$ & $112.29(17)$ & $\mathrm{C} 16-\mathrm{C} 17^{\prime}-\mathrm{H} 17 \mathrm{E}$ & 109.5 \\
\hline $\mathrm{C} 12-\mathrm{C} 11-\mathrm{H} 11 \mathrm{~A}$ & 109.1 & $\mathrm{H} 17 \mathrm{D}-\mathrm{C} 17^{\prime}-\mathrm{H} 17 \mathrm{E}$ & 109.5 \\
\hline $\mathrm{C} 3-\mathrm{C} 11-\mathrm{H} 11 \mathrm{~A}$ & 109.1 & $\mathrm{C} 16-\mathrm{C} 17^{\prime}-\mathrm{H} 17 \mathrm{~F}$ & 109.5 \\
\hline $\mathrm{C} 12-\mathrm{C} 11-\mathrm{H} 11 \mathrm{~B}$ & 109.1 & $\mathrm{H} 17 \mathrm{D}-\mathrm{C} 17^{\prime}-\mathrm{H} 17 \mathrm{~F}$ & 109.5 \\
\hline $\mathrm{C} 3-\mathrm{C} 11-\mathrm{H} 11 \mathrm{~B}$ & 109.1 & $\mathrm{H} 17 \mathrm{E}-\mathrm{C} 17^{\prime}-\mathrm{H} 17 \mathrm{~F}$ & 109.5 \\
\hline $\mathrm{C} 16-\mathrm{O} 2-\mathrm{C} 2-\mathrm{C} 3$ & $-112.3(2)$ & $\mathrm{O} 1-\mathrm{C} 1-\mathrm{C} 9-\mathrm{C} 10$ & $-175.23(19)$ \\
\hline $\mathrm{C} 16-\mathrm{O} 2-\mathrm{C} 2-\mathrm{C} 1$ & $71.8(2)$ & $\mathrm{C} 2-\mathrm{C} 1-\mathrm{C} 9-\mathrm{C} 10$ & $6.1(3)$ \\
\hline $\mathrm{O} 1-\mathrm{C} 1-\mathrm{C} 2-\mathrm{C} 3$ & $178.0(2)$ & $\mathrm{C} 6-\mathrm{C} 5-\mathrm{C} 10-\mathrm{C} 9$ & $1.0(3)$ \\
\hline
\end{tabular}




\begin{tabular}{llll}
$\mathrm{C} 9-\mathrm{C} 1-\mathrm{C} 2-\mathrm{C} 3$ & $-3.2(3)$ & $\mathrm{C} 6-\mathrm{C} 5-\mathrm{C} 10-\mathrm{C} 4$ & $-178.70(18)$ \\
$\mathrm{O} 1-\mathrm{C} 1-\mathrm{C} 2-\mathrm{O} 2$ & $-6.2(3)$ & $\mathrm{C} 8-\mathrm{C} 9-\mathrm{C} 10-\mathrm{C} 5$ & $-1.5(3)$ \\
$\mathrm{C} 9-\mathrm{C} 1-\mathrm{C} 2-\mathrm{O} 2$ & $172.54(16)$ & $\mathrm{C} 1-\mathrm{C} 9-\mathrm{C} 10-\mathrm{C} 5$ & $177.96(17)$ \\
$\mathrm{O} 2-\mathrm{C} 2-\mathrm{C} 3-\mathrm{C} 4$ & $-178.93(16)$ & $\mathrm{C} 8-\mathrm{C} 9-\mathrm{C} 10-\mathrm{C} 4$ & $178.22(17)$ \\
$\mathrm{C} 1-\mathrm{C} 2-\mathrm{C} 3-\mathrm{C} 4$ & $-3.4(3)$ & $\mathrm{C} 1-\mathrm{C} 9-\mathrm{C} 10-\mathrm{C} 4$ & $-2.3(3)$ \\
$\mathrm{O} 2-\mathrm{C} 2-\mathrm{C} 3-\mathrm{C} 11$ & $4.0(3)$ & $\mathrm{O} 4-\mathrm{C} 4-\mathrm{C} 10-\mathrm{C} 5$ & $-4.1(3)$ \\
$\mathrm{C} 1-\mathrm{C} 2-\mathrm{C} 3-\mathrm{C} 11$ & $179.59(18)$ & $\mathrm{C} 3-\mathrm{C} 4-\mathrm{C} 10-\mathrm{C} 5$ & $175.37(17)$ \\
$\mathrm{C} 2-\mathrm{C} 3-\mathrm{C} 4-\mathrm{O} 4$ & $-173.37(18)$ & $\mathrm{O} 4-\mathrm{C} 4-\mathrm{C} 10-\mathrm{C} 9$ & $176.20(18)$ \\
$\mathrm{C} 11-\mathrm{C} 3-\mathrm{C} 4-\mathrm{O} 4$ & $3.8(3)$ & $\mathrm{C} 3-\mathrm{C} 4-\mathrm{C} 10-\mathrm{C} 9$ & $-4.4(3)$ \\
$\mathrm{C} 2-\mathrm{C} 3-\mathrm{C} 4-\mathrm{C} 10$ & $7.2(3)$ & $\mathrm{C} 2-\mathrm{C} 3-\mathrm{C} 11-\mathrm{C} 12$ & $88.5(2)$ \\
$\mathrm{C} 11-\mathrm{C} 3-\mathrm{C} 4-\mathrm{C} 10$ & $-175.65(17)$ & $\mathrm{C} 4-\mathrm{C} 3-\mathrm{C} 11-\mathrm{C} 12$ & $-88.5(2)$ \\
$\mathrm{C} 10-\mathrm{C} 5-\mathrm{C} 6-\mathrm{C} 7$ & $0.3(3)$ & $\mathrm{C} 3-\mathrm{C} 11-\mathrm{C} 12-\mathrm{C} 13$ & $118.3(2)$ \\
$\mathrm{C} 5-\mathrm{C} 6-\mathrm{C} 7-\mathrm{C} 8$ & $-1.1(3)$ & $\mathrm{C} 11-\mathrm{C} 12-\mathrm{C} 13-\mathrm{C} 15$ & $178.2(2)$ \\
$\mathrm{C} 6-\mathrm{C} 7-\mathrm{C} 8-\mathrm{C} 9$ & $\mathrm{C} 11-\mathrm{C} 12-\mathrm{C} 13-\mathrm{C} 14$ & $-1.0(4)$ \\
$\mathrm{C} 7-\mathrm{C} 8-\mathrm{C} 9-\mathrm{C} 10$ & $0.6(3)$ & $\mathrm{C} 2-\mathrm{O} 2-\mathrm{C} 16-\mathrm{O} 3$ & $9.9(3)$ \\
$\mathrm{C} 7-\mathrm{C} 8-\mathrm{C} 9-\mathrm{C} 1$ & $0.7(3)$ & $\mathrm{C} 2-\mathrm{O} 2-\mathrm{C} 16-\mathrm{C} 17^{\prime}$ & $-170.81(18)$ \\
$\mathrm{O} 1-\mathrm{C} 1-\mathrm{C} 9-\mathrm{C} 8$ & $-178.78(19)$ & $\mathrm{C} 2-\mathrm{O} 2-\mathrm{C} 16-\mathrm{C} 17$ & $-170.81(18)$ \\
$\mathrm{C} 2-\mathrm{C} 1-\mathrm{C} 9-\mathrm{C} 8$ & $4.2(3)$ & & \\
\hline
\end{tabular}

Hydrogen-bond geometry $\left(A,{ }^{\circ}\right)$

\begin{tabular}{lllll}
\hline$D-\mathrm{H} \cdots A$ & $D-\mathrm{H}$ & $\mathrm{H} \cdots A$ & $D \cdots A$ & $D-\mathrm{H} \cdots A$ \\
\hline $\mathrm{C} 11-\mathrm{H} 11 B \cdots \mathrm{O} 4^{\mathrm{i}}$ & 0.97 & 2.55 & $3.274(3)$ & 131 \\
$\mathrm{C} 15-\mathrm{H} 15 A \cdots \mathrm{O} 1^{\mathrm{ii}}$ & 0.96 & 2.59 & $3.485(3)$ & 156 \\
\hline
\end{tabular}

Symmetry codes: (i) $-x+1 / 2, y-1 / 2,-z+3 / 2$; (ii) $x+1 / 2,-y+1 / 2, z+1 / 2$. 